\title{
Delay Dynamics of Cancer and Immune Cell Model
}

\author{
D. Adongo and K. R. Fister * \\ Department of Mathematics and Statistics, Murray State University, Murray, KY 42071, USA
}

\begin{abstract}
We investigate optimal control of a cancer-immune cell interactive model with delay in the interphase compartment. By applying the optimal control theory, we seek to minimize the cost associated with the chemotherapy drug, minimize the accumulation of cancer cells, and increase the immune cell presence. Optimality conditions and characterization of the control are provided. Numerical analyses are given to enhance the understanding of the difficulties that occur in the control of cancer.
\end{abstract}

Key words: optimal control, chemotherapy drug, cancer cells

AMS subject classification: 49J15, 93C15

\section{Introduction}

Cells that can not regulate their own growth and division are classified as cancerous. Lu et al. [12] paper takes a more extensive look at the development of cancer cells by noting the three phases through which they travel. These stages are the mitotic-phase (dividing), quiescent phase (resting), and the interphase. The body's main defenses against tumors are the white blood cells, often called lymphocytes, generated by the immune system. Numerous variables, such as toxicity, cause treatment to be highly complex. This complexity causes the strategy of treatment to vary widely from person to person. Immunotherapy is also another way to fight cancer. This type of treatment is a process of boosting the immune system in specific areas which target cancer cells and leave healthy cells relatively untouched. While both treatments are usually used separately, Kirschner and Panetta [15] investigate the biological perspective of a combination therapy approach. This research has sparked interest in theoretical exploration using mathematical modeling. Recent work

${ }^{*}$ Corresponding author. E-mail: renee.fister@murraystate.edu 
by dePillis et al. and Ledzewicz [16, 17] provide guidance in the treatment of cancers using a mixed strategy of chemotherapy and immunotherapy in conjunction with optimal control techniques.

It can be quite useful to use mathematical modeling when determining strategies for fighting diseases which will enhance the quality of life for the patients. By using updated mathematical models, it is possible to make quantitative and testable predictions about real life patients. This enables a more accurate approach to treatments [18]. With the collaboration among biologists, mathematicians, and medical professionals, mathematical concepts that were more engineering based have been found to be useful in medical applications, $[6,7,8,22,9]$. The goal of applying optimal control theory to mathematical models representing the interaction between tumor, immune system, and chemotherapy is to determine the ideal mix of treatments that minimizes both tumor mass and negative effects upon the health of the patient. A study from 2000 uses optimal control techniques in designing drug protocols that will kill a desired amount of tumor cells without killing the host, [1]. Works by Kim et al. [14], Swan and Vincent [21], and Murray [19] have successfully applied optimal control to maximize the effects of the chemotherapy drug while minimizing the toxicity and damage done by the drug.

A recent study by Lu et al. [12] looks at the stability of a mitotic phase-specific drug and its interaction with the immune system and cancer cells, whose results show a significant decrease in the number of cancer cells but no change in the stability. Another study by Villasana and Radunskaya [23] also considered the stability of using a cycle-specific drug and found that the stability depended on a delay of the cells moving from the interphase to mitosis. It is important to note that [23] didn't look at the quiescent phase; whereas, [12] found that this phase greatly influences the cancer as a whole.

This paper is organized as follows. Section 2 gives a discussion of the model, a description of the objective functionals, and the characterization of the optimal controls. Section 3 describes numerical simulations and graphical depictions of the optimal control regimens. Section 4 provides summary remarks.

\section{Model}

The cell cycle is organized into 3 phases; the quiescent phase where cells rest, the interphase where cells prepare for mitosis, and then the mitotic phase where cells divide. Lu et al. [12] develops the interaction between cells and the three phases, the immune system, and the cyclespecific drug. The variables used are as follows:

- $x$-number of cancer cells in interphase phase

- $y$ - number of cancer cells in mitotic phase

- $z$-number of cancer cells in the quiescent phase

- $I$ - number of lymphocytes 
- $u$ - biomass of chemotherapy drug

The model is:

$$
\begin{aligned}
\frac{d x}{d t}= & s \alpha_{3} z(t)-\alpha_{1} x(t)-\left(\sigma_{1}+k_{1} I(t)\right) x(t) \\
\frac{d y}{d t}= & \alpha_{1} x(t-\tau)-\left(\alpha_{2}+\sigma_{2}+k_{2} I(t)\right) y(t)-k_{4}\left(1-\left(e^{-k_{5} u(t)}\right) y(t)\right. \\
\frac{d z}{d t}= & 2 s^{-1} \alpha_{2} y(t)-\left(\alpha_{3}+\sigma_{3}+k_{3} I(t)\right) z(t) \\
\frac{d I}{d t}= & k+\left(\frac{\rho I(t)(x+y+s z)^{n}}{a+(x+y+s z)^{n}}\right)-\left(\sigma_{4}+c_{1} x(t)+c_{2} y(t)+c_{3} z(t)\right) I(t) \\
& -k_{6}\left(1-e^{-k_{7} u(t)}\right) I(t) \\
\frac{d u}{d t}= & -\gamma u(t)+v(t)
\end{aligned}
$$

where the initial conditions are

$$
x(t)=\phi_{1}, t \in[-\tau, 0], y(0)=y_{0}, z(0)=z_{0}, I(0)=I_{0}, u(0)=u_{0}
$$

It is important to note that all the constants are positive and that differential equation describing the mitotic phase is the only one with a delay present. The terms $\alpha_{1}, \alpha_{2}, \alpha_{3}$ describe the rate at which cells travel from each phase. The natural death rate of cells have parameters denoted by $\sigma_{1}, \sigma_{2}, \sigma_{3}$, while the death rate parameters by the immune cells are given as $c_{1}, c_{2}, c_{3}$. The term $\frac{\rho I(t)(x+y+s z)^{n}}{a+(x+y+s z)^{n}}$ is the nonlinear growth of the immune system [23]. Destruction by drugs is shown by $\left(1-e^{-k_{5} u(t)}\right)$ and $\left(1-e^{-k_{7} u(t)}\right)$ [23]. We assume that once the chemotherapy drug is given, it has an exponential decay rate of $-\gamma$. The control function is given by $v(t)$. We also assume that $u_{0}>1$.

\subsection{Objective Functional}

The general form of the objective functionals incorporates quadratic control. Since this is a first attempt at understanding the dynamics of the model in relation to optimal control with numerical descriptions, the mathematical intricacies with a nonlinear control term are less varied. In a future work, incorporation of a linear control situation will be assessed. For some generality, we include a set of objective functionals without salvage terms and the other with salvage terms. We seek to minimize all the objective functionals over our class of controls, $\mathbb{V}=\left\{t \in\left[0, t_{f}\right] \mid 0 \leq v(t) \leq 1\right\}$. Here $\epsilon$ is a weight factor, representing the cost to the system and $x, y, z$ are the cancer cells in specific phases of the cell cycle. We are minimizing both the tumor cells and the cost associated with the chemotherapy drug. The weight parameters $C, D, E$, and $F$ are inserted to allow for discussion of the importance of the minimization strategy as these constants are varied.

$$
J_{1}(v)=\int_{0}^{t_{f}}\left[\frac{\epsilon}{2} v^{2}(t)+D x(t)+C y(t)+E z(t)-F I(t)\right] d t
$$


Table 1: Meaning of parameters for system (2.1-2.6)

\begin{tabular}{|c|c|}
\hline Parameter & Parameter description \\
\hline$\alpha_{1}$ & rate at which cells move into mitosis \\
\hline$\alpha_{2}$ & rate at which cells move into the quiescent phase \\
\hline$\alpha_{3}$ & rate cells leave quiescent and enter cell cycle \\
\hline$c_{i}$ for $i=1,2,3$ & binding losses with immune cells \\
\hline$\sigma_{i}$ for $i=1,2,3,4$ & natural death rates for $x, y, z$, and $I$ \\
\hline$\rho$ & proportional growth of $I$ due to interaction with cancer cells \\
\hline$n$ & fractional exponent of growth from stimulus of cancer cells \\
\hline$a$ & rate at which $I$ reach saturation without stimulus \\
\hline$k$ & growth rate of $I$ with no cancer cells \\
\hline$k_{i}$ for $i=1,2,3$ & rate $I$ destroys cells in different phases \\
\hline$k_{4}, k_{6}$ & rate of removal of $y$ and $I$ \\
\hline$k_{5}, k_{7}$ & rate of drugs in removal of $y$ and $I$ \\
\hline$\gamma$ & natural decay rate of chemotherapy \\
\hline$\tau$ & time of cells in interphase (delay variable) \\
\hline
\end{tabular}

over the set $\mathbb{V}=\left\{t \in\left[0, t_{f}\right] \mid 0 \leq v(t) \leq 1\right\}$. Here $\epsilon$ is a weight factor, representing the cost to the system and $x, y, z$ are the cancer cells. So we are minimizing both the tumor cells and the cost associated with the chemotherapy drug. We will also minimize the second objective functional

$$
J_{2}(v)=\int_{0}^{t_{f}} \frac{\epsilon}{2} v^{2}(t) d t+\left[x\left(t_{f}\right)+y\left(t_{f}\right)+s z\left(t_{f}\right)\right]
$$

over the same set and with the same weight factor $\epsilon$. The cost associated with drug is being minimized, but the cancer cells $x, y$, and $z$ are minimized at the final time.

As we have proven the existence of the solution to the system given the control and developed the optimality conditions in [4], we reiterate the conditions here and move toward the discussion of the numerical situations.

\subsection{Characterization of the Controls}

Given that an optimal control exists, we can depict the characterization of the optimal control using a version of Pontryagin's Maximum Principle applicable to delay situations, [20].

We first define the two Hamiltonians, $H_{1}$, which is associated with the objective functional 
$J_{1}(v)$, and $H_{2}$, which is associated with the second objective functional $J_{2}(v)$.

$$
\begin{aligned}
H_{1}= & D x(t)+C y(t)+E z(t)-F I(t)+\frac{\epsilon}{2} v^{2}(t) \\
& +\lambda_{1}\left[\left(-\left(\alpha_{1}+\sigma_{1}\right) x(t)+s \alpha_{3} z(t)-k_{1} x(t) I(t)\right)\right] \\
& +\lambda_{2}\left[\left(\alpha_{1} x(t-\tau)-\left(\alpha_{2}+\sigma_{2}\right) y(t)-k_{2} y(t) I(t)-k_{4}\left(1-e^{-k_{5} u(t)}\right) y(t)\right)\right] \\
& +\lambda_{3}\left[2 s^{-1} \alpha_{2} y(t)-\left(\alpha_{3}+\sigma_{3}\right) z(t)-k_{3} z(t) I(t)\right] \\
& \left.+\lambda_{4}\left[k+\frac{\rho I(t)(x+y+s z)^{n}}{\left(a+(x+y+s z)^{n}\right)}-\left(\sigma_{4}+c_{1} x(t)+c_{2} y(t)+c_{3} z(t)\right) I(t)-k_{6}\left(1-e^{-k_{7} u(t)}\right) I(t)\right)\right] \\
& +\lambda_{5}[-\gamma u(t)+v(t)]
\end{aligned}
$$

and

$$
\begin{aligned}
H_{2}= & \frac{\epsilon}{2} v^{2}(t) \\
& +\lambda_{1}\left[\left(-\left(\alpha_{1}+\sigma_{1}\right) x(t)+s \alpha_{3} z(t)-k_{1} x(t) I(t)\right)\right] \\
& +\lambda_{2}\left[\left(\alpha_{1} x(t-\tau)-\left(\alpha_{2}+\sigma_{2}\right) y(t)-k_{2} y(t) I(t)-k_{4}\left(1-e^{-k_{5} u(t)}\right) y(t)\right)\right] \\
& +\lambda_{3}\left[2 s^{-1} \alpha_{2} y(t)-\left(\alpha_{3}+\sigma_{3}\right) z(t)-k_{3} z(t) I(t)\right] \\
& \left.+\lambda_{4}\left[k+\frac{\rho I(t)(x+y+s z)^{n}}{\left(a+(x+y+s z)^{n}\right)}-\left(\sigma_{4}+c_{1} x(t)+c_{2} y(t)+c_{3} z(t)\right) I(t)-k_{6}\left(1-e^{-k_{7} u(t)}\right) I(t)\right)\right] \\
& +\lambda_{5}[-\gamma u(t)+v(t)]
\end{aligned}
$$

Theorem 1. Given an optimal control, $v^{*}(t)$, and solutions of the corresponding state system, there exist adjoint variables $\lambda_{i}$ for $i=1,2, \ldots 5$ satisfying the following:

$$
\begin{array}{r}
-\frac{\partial H}{\partial x}-\left.\frac{\partial H}{\partial x(t-\tau)}\right|_{t+\tau}=\lambda_{1}^{\prime}=-D+\lambda_{1}\left(\alpha_{1}+\sigma_{1}\right)+\lambda_{1} k_{1} I-\lambda_{4} \frac{\rho \operatorname{Ina}(x+y+s z)^{(n-1)}}{\left(a+(x+y+s z)^{n}\right)^{2}} \\
+\quad \lambda_{4} c_{1} I-\left.\lambda_{2} \alpha_{2}\right|_{t+\tau} \\
\quad \text { for } 0 \leq t \leq t_{f}-\tau \\
-\frac{\partial H}{\partial x_{t}}=\lambda_{1}^{\prime}=-D+\lambda_{1}\left(\alpha_{1}+\sigma_{1}\right)+\lambda_{1} k_{1} I-\lambda_{4} \frac{\rho \operatorname{Ina}(x+y+s z)^{(n-1)}}{\left(a+(x+y+s z)^{n}\right)^{2}}+\lambda_{4} c_{1} I \quad \\
\quad \text { for } t_{f}-\tau \leq t \leq t_{f}
\end{array}
$$


For the last four adjoints, the interval for $t$ is $\left[0, t_{f}\right]$.

$$
\begin{aligned}
-\frac{\partial H}{\partial y} & =\lambda_{2}^{\prime}=-C+\lambda_{2} k_{2} I+\lambda_{2} k_{4}\left(1-e^{-k_{5} u}\right)+\lambda_{2}\left(\alpha_{2}+\sigma_{2}\right)-\lambda_{3}\left(2 s^{-1} \alpha_{2}\right) \\
& -\lambda_{4}\left(\left(\frac{a \rho I(t) n(x+y+s z)^{(n-1)}}{\left(a+(x+y+s z)^{n}\right)^{2}}\right)-c_{2} I\right) \\
-\frac{\partial H}{\partial z} & =\lambda_{3}^{\prime}=-E-\lambda_{1} s \alpha_{3}+\lambda_{3}\left(\alpha_{3}+\sigma_{3}+I(t) k_{3}\right) \\
& -\lambda_{4}\left(\frac{a \rho I(t) n a s(x+y+s z)^{(n-1)}}{\left(a+(x+y+s z)^{n}\right)^{2}}\right)-\lambda_{4} c_{3} I(t) \\
-\frac{\partial H}{\partial I} & =\lambda_{4}^{\prime}=F+\lambda_{1} k_{1} x+\lambda_{2} k_{2} y+\lambda_{3} k_{3} z \\
& -\lambda_{4}\left(\left(\frac{\rho(x+y+s z)^{n}}{a+(x+y+s z)^{n}}+\sigma_{4}+c_{1} x+c_{2} y+c_{3} z+k_{6}\left(1-e^{-k_{7} u}\right)\right)\right) \\
-\frac{\partial H}{\partial u} & =\lambda_{5}^{\prime}=\lambda_{2} k_{4} k_{5} e^{-k_{5} u(t)} y+\lambda_{4} k_{6} k_{7} e^{-k_{7} u(t)} I+\lambda_{5} \gamma
\end{aligned}
$$

with $\lambda_{i}\left(t_{f}\right)=0$ for $i=1,2, . .5$.

We note that the adjoint equations associated with the objective functional $J_{2}(v)$ are the same as for equations (2.10)-(2.15). However, the transversality conditions are $\lambda_{i}\left(t_{f}\right)=1$ for $i=1,2,3$ and $\lambda_{j}\left(t_{f}\right)=0$ for $j=4,5$. Furthermore, $v^{*}(t)$ associated with either $J_{1}(v)$ or $J_{2}(v)$ can be represented by

$$
v^{*}(t)=\min \left(\max \left(0, \frac{-\lambda_{5}(t)}{\epsilon}\right), 1\right)
$$

\section{Numerical Simulations for Quadratic Control}

The two systems of ODEs were solved numerically using the classical fourth-order Runge-Kutta method [3]. The system (2.1-2.6) is solved forward in time with the state variables being stored in the vectors $\mathbf{x}, \mathbf{y}, \mathbf{z}, \mathbf{I}$ and $\mathbf{u}$. The system comprising of adjoints (2.10-2.15) is solved backward in time since the final time conditions for the adjoints are given. The adjoint variables are stored in the vectors $\lambda_{1}, \lambda_{2}, \lambda_{3}, \lambda_{4}$, and $\lambda_{5}$. The following algorithm was followed

- An initial guess for $\mathbf{v}$ was made.

- Using the initial conditions and the values for $\mathbf{v}$, the quantities $\mathbf{x}, \mathbf{y}, \mathbf{z}, \mathbf{I}$ and $\mathbf{u}$ are solved for.

- Using the final conditions for the adjoints and the values of $\mathbf{x}, \mathbf{y}, \mathbf{z}, \mathbf{I}$ and $\mathbf{u}$, the adjoints $\boldsymbol{\lambda}_{1}$, $\lambda_{2}, \lambda_{3}, \lambda_{4}$, and $\lambda_{5}$ are computed. 
- The new value for $\mathbf{v}$ is obtained from (2.16).

- Stop if for all the computed values the relative error is less than some prescribed small number tol in some norm.

$$
\frac{\|(\text { new value })-(\text { old value }) \|_{\infty}}{\|(\text { new value }) \|_{\infty}}<\text { tol }
$$

Otherwise go back to the second step.

For the parameter data see Table 2 in Appendix. For the function $\phi_{1}$ in the initial conditions (2.7) the following were considered:

1. exponential function

$$
\phi_{1}(t)=x_{0} e^{\alpha_{1} t}
$$

2.

$$
\phi_{1}(t)=\frac{t}{K+t}
$$

where $K$ is chosen as half of the initial value or half of value $x(t)$ without drug.

Next, we provide scenarios with the parameter sets displayed in the Appendix to illustrate the ability to control the progression of the cancer cells. In some instances, we see that with maximum drug, the cancer growth dominates. However, we do find success with the inclusion of the immune component. Utilization of the two objective functions and the parameter sets give both discouraging and encouraging results as discussed below. All the given figures correspond to $\phi_{1}(t)$ being the exponential function, since this gives us the most realistic results.

\subsection{Case $J_{1}(v)$}

Consider the first objective functional given by (2.8). Using the initial conditions $x(0)=y(0)=$ $1 \times 10^{5}, z(0)=2 \times 10^{5}$ and $u(0)=8$ with the cost to the system, $\epsilon=100$, the number of cancer cells and control function for Data I are given in Figure 1. The weight parameters used in Figures 1 and 2 are $C=D=1, E=z(0) / x(0)$, and $F=0$.

We choose $F=0$ in the objective functional to see the interplay in the cancer phases in the minimization procedure without the maximization of the immunological component. If we allow the lymphocytes to interact in the model without giving it emphasis in the objective functional, we notice the need to give maximal drug and find that one cannot control the cancer. Other following situations with the inclusion of the this weight factor, $F$ are provided in connection with Figure 4. 

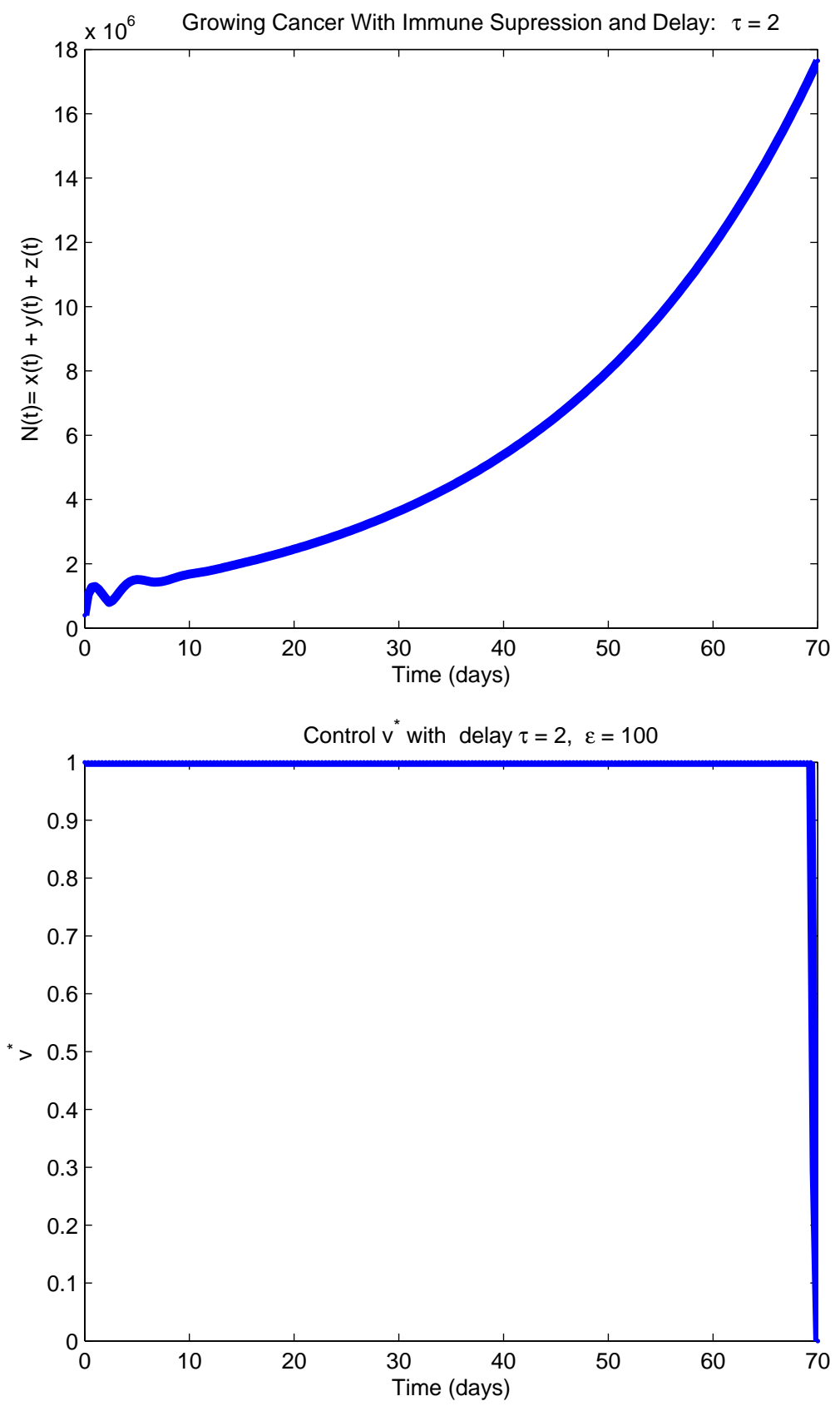

Figure 1: Number of cancer cells and control $v$

Simulating the experiment over a period of 70 days, it is observed that the control pushes $u(t)$ constantly up before dropping to zero very sharply at the end. Note that from (2.6), $u(t)$ decreases monotonically if $v(t)$ is absent. We note that giving the same emphasis to controlling the cancer cells in the interphase and mitotic phase with no importance given to the increase of the lymphocytes in our objective functional does not improve our chances of controlling the total cancer cells even though maximum drug is given for almost the entire two month period. 

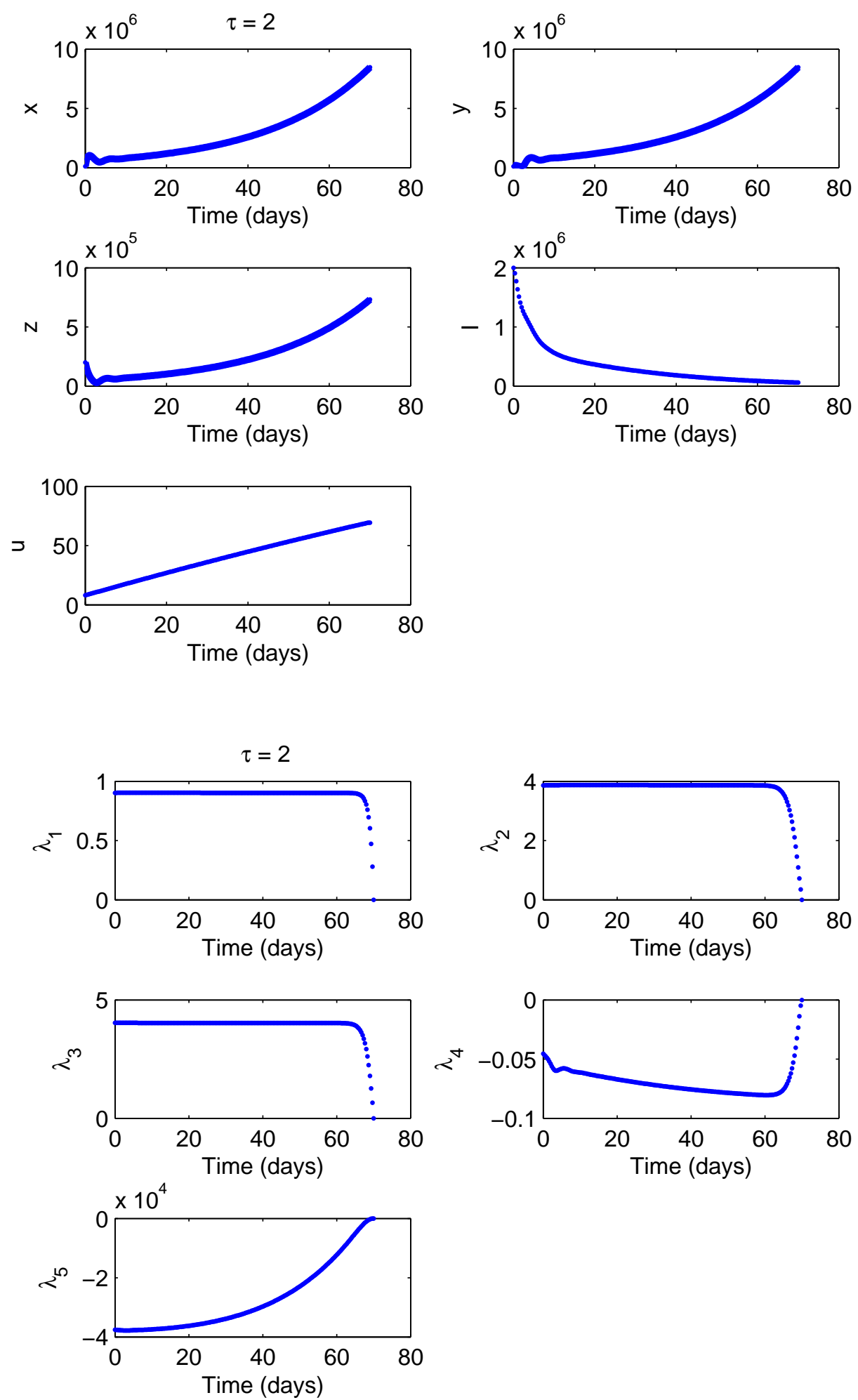

Figure 2: State and adjoint variables 
In Figure 2, the adjoint, state, and control functions are graphed to allow the reader to see the intricacies between the variables, specifically, $\lambda_{5}(t)$ and $u(t)$ through the control $v(t)$. We see that the immune component as portrayed in the lymphocytes are decaying. We can surmise that the body ins not able to regenerate those cells to mitigate the negative effects of the cancer cells.

We see that we have better results with using data II from the Appendix with the same time delay. Sample graphs of the number of cancer cells and control function using data II are given in Figure 3
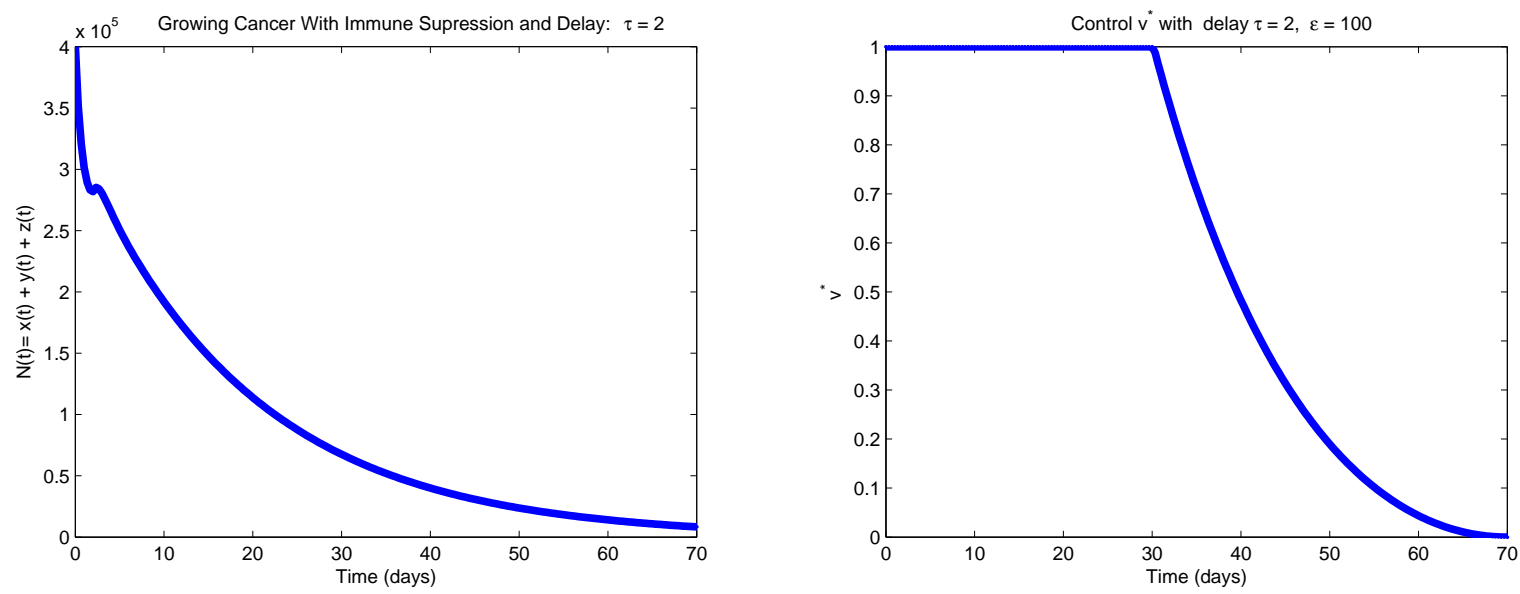

Figure 3: Number of cancer cells and control $v$

In Figure 3, we see that the same weight factors as in Figure 1 but with different transition rates through the cell cycle and with marginally different death rates leads to reduction of the cancer cells with considerably less amount of drug for a fixed delay.

As the parameter $F$, the weight factor related to the lymphocytes, is increased, it is observed that less control needs to be applied, see Figure 4. As F increases, the period over which the drug is administered decreases. This is consistent with the concept of availability of more lymphocytes to increase the body's natural immunity, [9]. Therefore, less drug is needed. The control is generally zero initially before rising sharply to a constant value before dropping off sharply back to zero close to the end.

On the other hand, if the other parameters, $C, D$ and $E$ are all zero, increasing $F$ does not have an effect on the control. The control is zero everywhere. The cancer cells decrease with the need for additional control. 

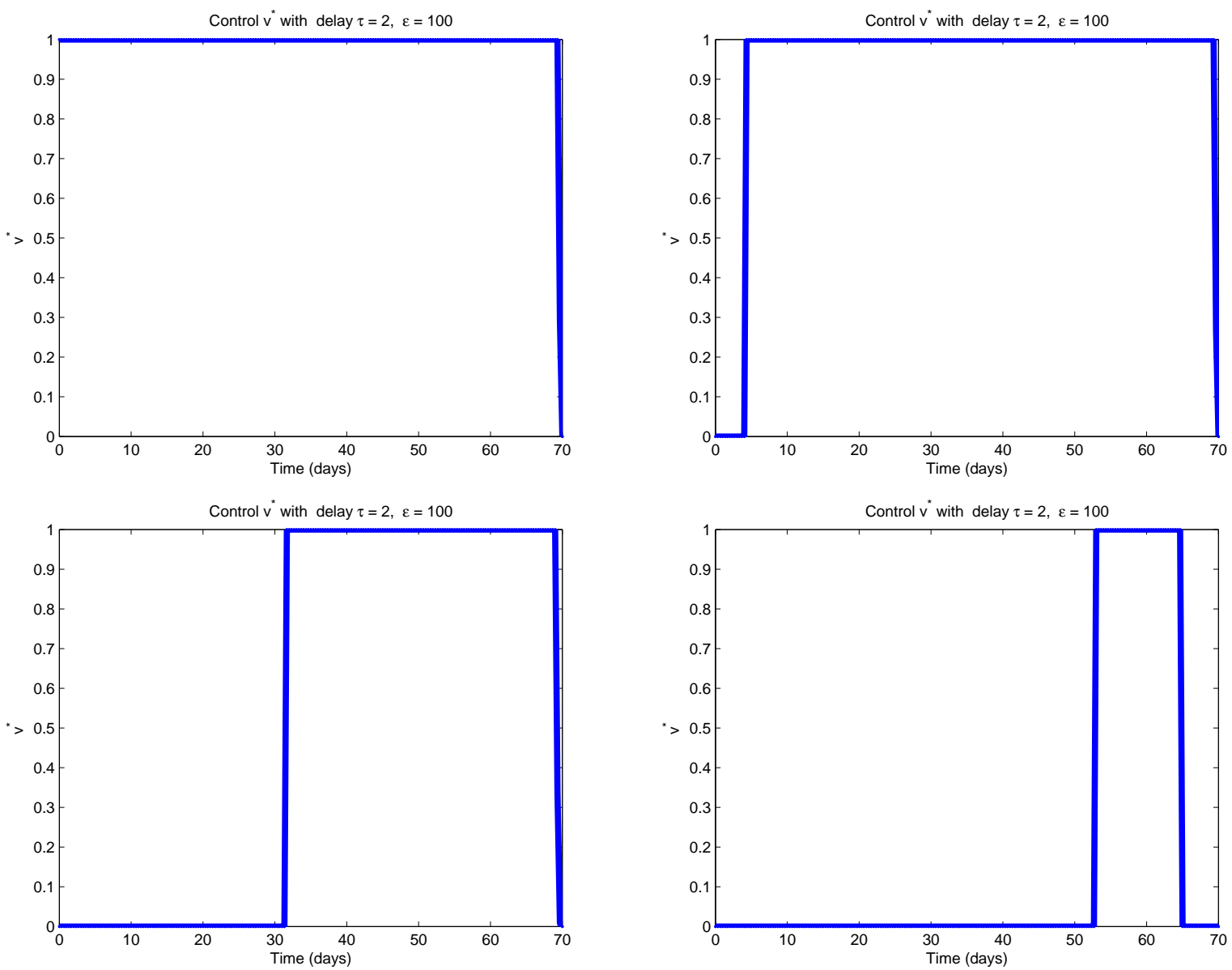

Figure 4: Control $v$ with $F=1,10,100,1000$

Increasing the parameter $C$, the weight factor associated with cells in mitotic phase, while keeping the other parameters zero has the effect of increasing the period over which control is applied, see Figure 5 obtained from Data III. The idea is that the emphasis is on minimizing the total number of cancer cells in the mitotic phase. Consequently, more treatment is needed to create a reduction in the total number of cancer cells. 

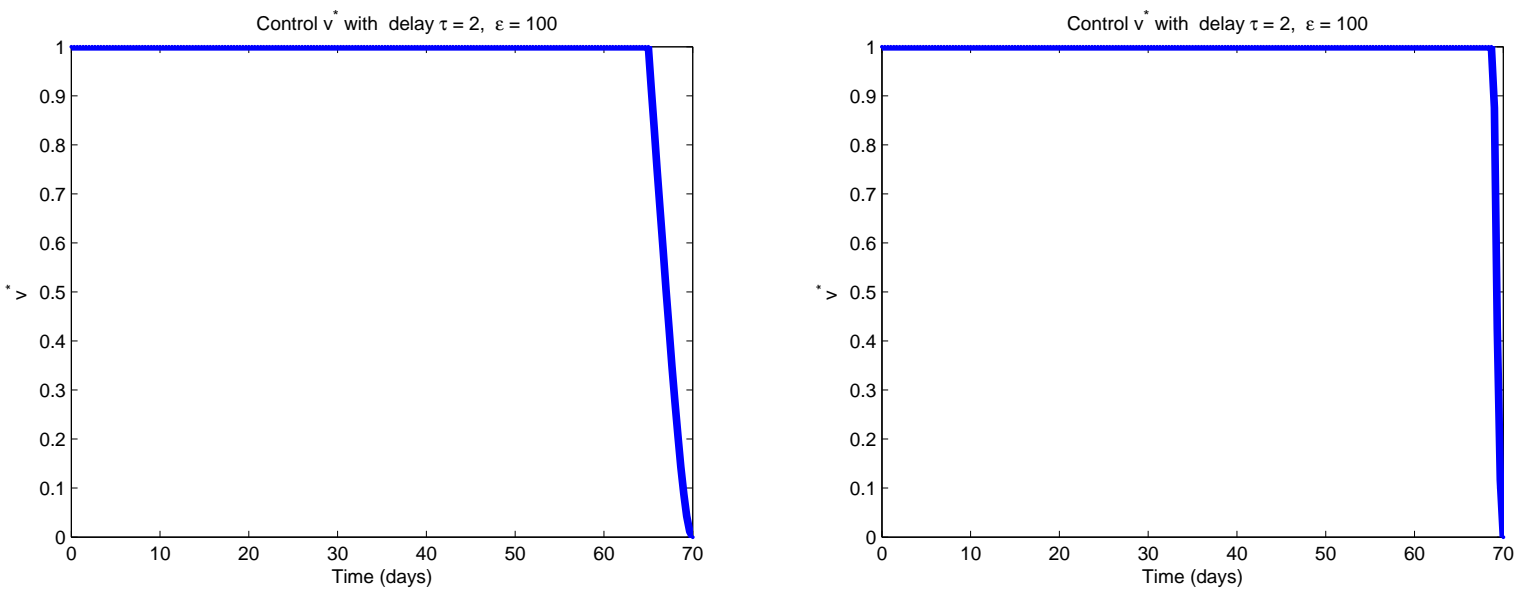

Figure 5: Control $v$ with $C=1,10$

In this section, we consider the objective functional that includes the minimization of the drug and the cancer cells at the final time. The concept of minimization of the cancer cells at the final time is included as this salvage term. Clinically, tumors are usually evaluated at the end of a treatment regimen. Therefore, this situation is analyzed.

\subsection{Case $J_{2}(v)$}

In this section, we consider the objective functional that includes the minimization of the drug and the cancer cells at the final time. The concept of minimization of the cancer cells at the final time is included as this salvage term. Clinically, tumors are usually evaluated at the end of a treatment regimen. Therefore, this situation is analyzed.

The number of cancer cells and control function for Data I are given in Figure 6. The initial conditions for the state variables are the same as in the $J_{1}(v)$ case.

It is interesting that the predicted optimal control would be to give maximal drug, but the tumor is not controllable.

Figure 7 gives graphs of the number of cancer cells and control function for Data II. With the changes in the few parameters relating to the rate of movement of the cells and death rates, we have a very different schematic than in Figure 6. We can reduce the cancer volume and allow for less treatment.

Using Data III, it is observed that increasing the cost parameter, $\epsilon$, decreases the amount of control applied (see Figure 8). Please note that the qualitative nature of the control remains constant in the four graphics. Yet, the scale on the dosage amount is reduced by a factor of ten. For each $\epsilon$ the control increases at a constant rate, dropping of sharply at the end. The corresponding graphs of number of cancer cells are given in Figure 9.

As the cost parameter increases the number of cancer cells at the end of the time period decreases for a while. At some point, increasing the cost parameter leads to an increase in the number 

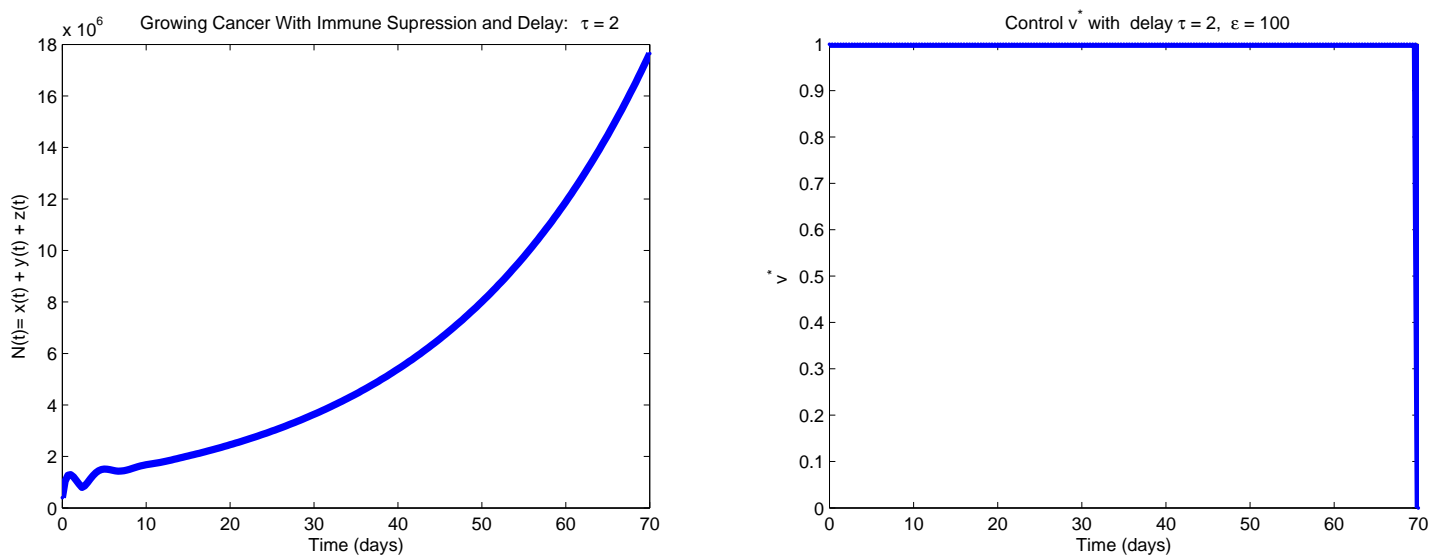

Figure 6: Number of cancer cells and control $v$ (Data I)
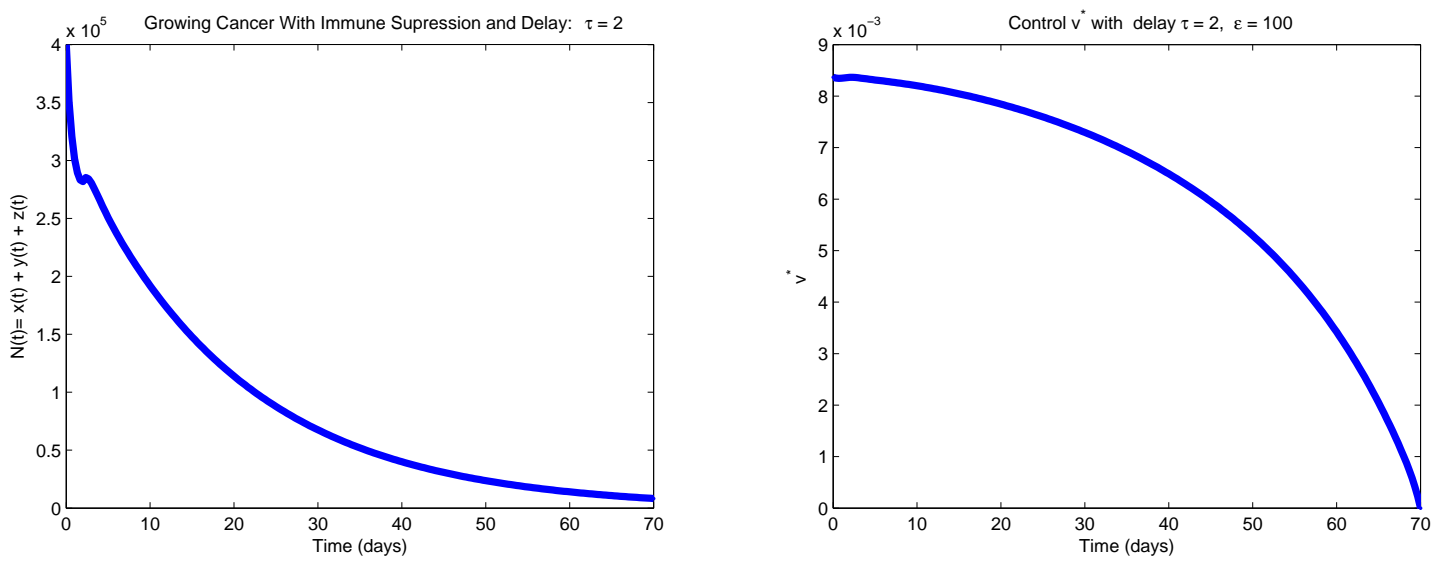

Figure 7: Number of cancer cells and control $v$ (Data II)

of cancer cells at the end but not to the values for the starting cost parameter $\epsilon=100$. There is a point at which the weight factor, $\epsilon$, creates little added benefit to the cancer reduction. Although the drug given is reduced, the cancer reaches a plateau of relatively stable setting. In the two month window, this could be viewed as a state of remission with a slight incline in the total cancer cells for the cases $\epsilon=1000,10000,100000$. 

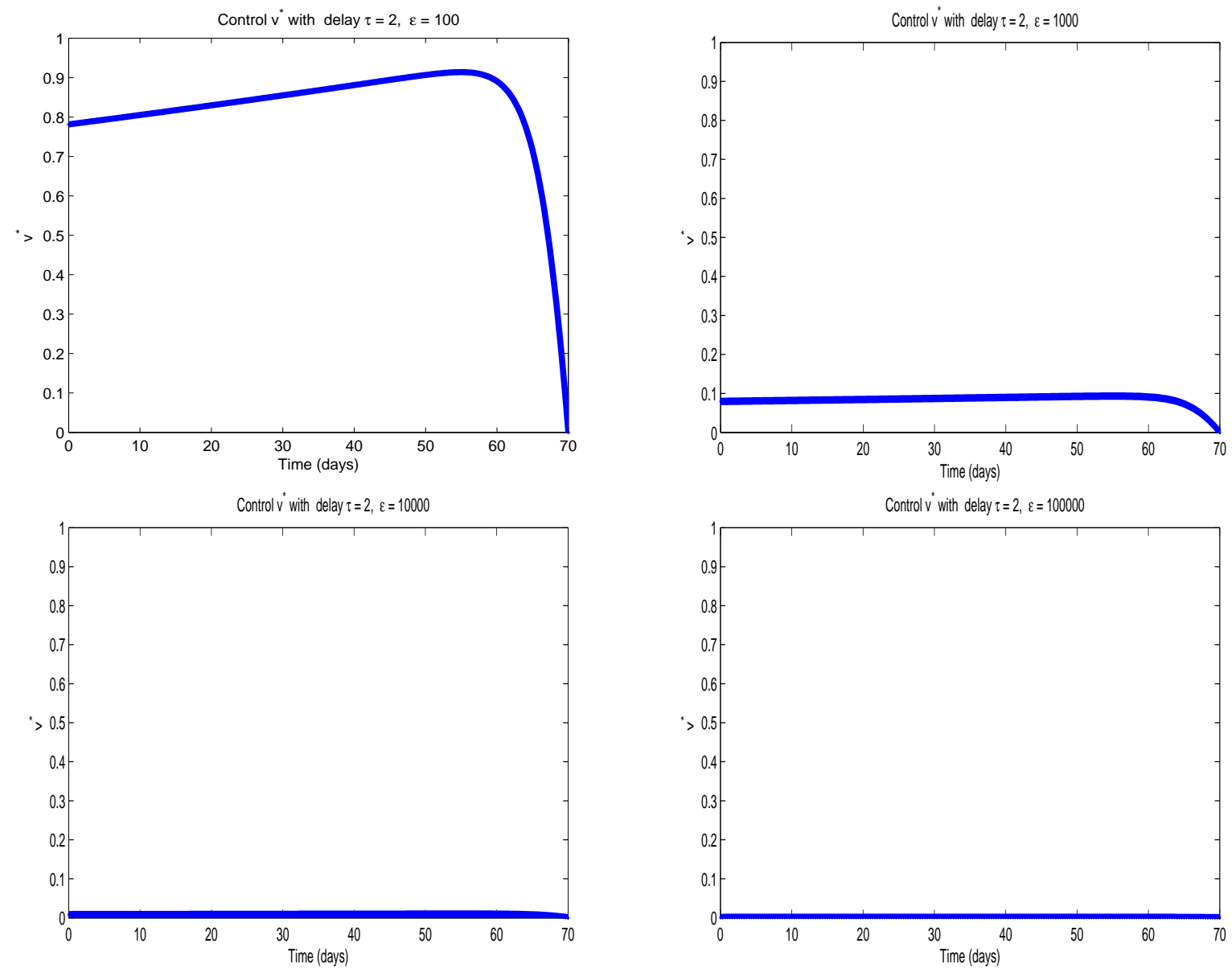

Figure 8: Control $v$ with varying $\epsilon$ 

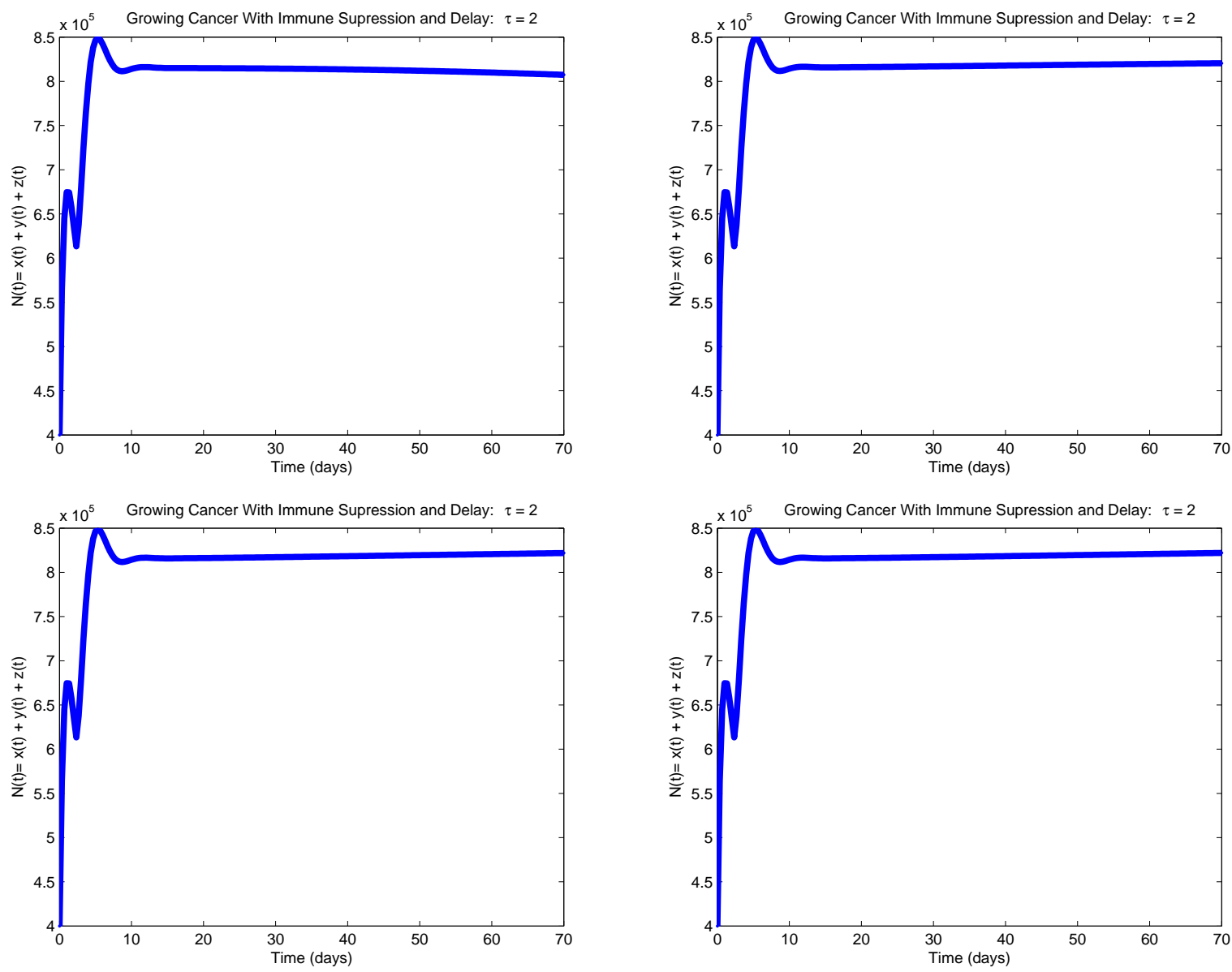

Figure 9: Number of cancer cells with varying $\epsilon$

\section{Conclusion}

The introduction of a drug treatment control can help reduce or stabilize the growth of cancer cells in its various stages of the cell cycle. The combination of delay, drug control, and cancer interactions with the immune cells create different scenarios based on the parameter set choices. The encouraging result is that changing the weight factor $F$ in the immune system produces the effect of reducing the drug needed to control the cancer growth. Further studies with variable delays and immune therapy may provide more information about the necessary medical strategy to eradicate the tumor. 


\section{Acknowledgements}

We would like to acknowledge the Murray State University BioMaPS program and the NSF grants DMS-0531865 and DUE-1028125 for support.

\section{Appendix}

The parameter data used in the numerical simulation are given in Table 2.

\begin{tabular}{|c|c|c|c|l|}
\hline Parameter & Data I & Data II & Data III & units \\
\hline$\alpha_{1}$ & 1.0 & 0.84 & 1.0 & per day \\
$\alpha_{2}$ & 0.6 & 0.9 & 0.6 & per day \\
$\alpha_{3}$ & 0.9 & 0.024 & 0.264 & per day \\
$c_{1}$ & $0.2 \mathrm{e}-6$ & & & per cell day \\
$c_{2}$ & $0.8 \mathrm{e}-7$ & & & per cell day \\
$c_{3}$ & $0.108 \mathrm{e}-6$ & & & per cell day \\
$\sigma_{1}$ & 0.11 & & & per day \\
$\sigma_{2}$ & 0.28 & 0.67 & 0.28 & per day \\
$\sigma_{3}$ & $0.1 \mathrm{e}-4$ & 0.056 & 0.056 & per day \\
$\sigma_{4}$ & 0.3 & & & per day \\
$\rho$ & 0.2 & & & per day \\
$a$ & $0.5 *(0.1 e 6)^{3}$ & & & (cell) \\
$k$ & $0.15 \mathrm{e} 6$ & & & cell per day \\
$k_{1}$ & $0.1 \mathrm{e}-7$ & & & per cell day \\
$k_{2}$ & $0.4 \mathrm{e}-8$ & & & per cell day \\
$k_{3}$ & $0.1 \mathrm{e}-8$ & & & per cell day \\
$k_{4}$ & 0.25 & & & per day \\
$k_{5}$ & $0.25 \mathrm{e}-3$ & & & per mg \\
$k_{6}$ & $0.3 \mathrm{e}-1$ & & & per day \\
$k_{7}$ & $0.5 \mathrm{e}-2$ & & & per mg \\
$\gamma$ & $0.3 \mathrm{e}-2$ & & & per day \\
$n$ & $\frac{3}{4}$ & & & \\
\hline
\end{tabular}

Table 2: The parameter values not given for data sets II and III use the values from data set I. 


\section{References}

[1] I. Athanassios, D. Barbolosi. Optimizing drug regimens in cancer chemotherapy by an efficacy-toxicity mathematical model. Compu. Biomedical Res., 33 (2000), 211-226.

[2] M. Chaplain, A. Matzavinos. Mathematical modelling of spatio-temporal phenomena in tumour immunology tutorials in mathematical biosciences III. Cell Cycle, Proliferation, and Cancer, (2006), 131-183.

[3] W. Cheney, D. Kincaid. Numerical mathematics and computing. Thomson Brooks/Cole, Belmont, 2008.

[4] C. Collins, K. R. Fister, M. Williams. Optimal control of a cancer cell model with delay. Math. Model. Nat. Phen., 5 (2010), No. 3, 63-71.

[5] P. C. Das, R. R. Sharma. On optimal controls for measure delay-differential equations. SIAM J. Control, 6 (1971), No. 1, 43-61.

[6] L. G. dePillis, A. E. Radunskaya. A mathematical tumor model with immune resistance and drug therapy: an optimal control approach. J. Theoretical Medicine, 3 (2001), 79-100.

[7] L. G. dePillis, A. E. Radunskaya. The dynamics of an optimally controlled tumor model: a case study. Math. Comp. Model., 37 (2003), No. 11, 1221-1244.

[8] L. G. dePillis, A. E. Radunskaya, C. L. Wiseman. A validated mathematical model of cellmediated immune response to tumor growth. Cancer Research, 61 (2005), No. 17, 79507958.

[9] L. G. dePillis, K. R. Fister, W. Gu, C. Collins, M. Daub, J. Moore, B. Preskill. Mathematical model creation for cancer chemo-immunotherapy. Computational and Math. Methods in Medicine, 10 (2009), No. 3, 165-184.

[10] R. D. Driver. Ordinary and delay differential equations. Springer-Verlag, New York, 1977.

[11] R. Fletcher. Practical methods of optimization. Wiley and Sons, New York, 1987.

[12] W. Lu, T. Hillen, H. I. Freedman. A mathematical model for M-phase specific chemotherapy including the $G_{o}$-phase and immunoresponse. Math. Biosci. and Engng.,4 (2007), No. 2, 239-259.

[13] M.I. Kamien, N. L. Schwartz. Dynamic optimization: the calculus of variations and optimal control in economics and management, Advanced Textbooks in Economics. North-Holland, 1991.

[14] M. Kim, S. Perry, K. B. Woo Quantitative approach to the design of antitumor drug dosage schedule via cell cycle kinetics and systems theory. Ann. Biomed. Engng, 5 (1977), 12-33. 
[15] D. Kirschner, J. C. Panetta. Modeling immunotherapy of the tumor-immune interaction. J. Math. Bio., 35 (1998), 235-252.

[16] U. Ledzewicz, T. Brown, H. Schattler. Comparison of optimal controls for a model in cancer chemotherapy with $l_{1}$ and $l_{2}$ type objectives. Optimization Methods and Software, 19 (2004), No. 3-4, 339-350.

[17] U. Ledzewicz, H. Schattler. Drug resistance in cancer chemotherapy as an optimal control problem. Discrete and Continuous Dynamical Systems - Series B, 6 (2006), No. 1, 129-150.

[18] D. McKenzie. Mathematical modeling and cancer. SIAM News, 31 (2004), 1-2.

[19] J. M. Murray. Some optimality control problems in cancer chemotherapy with a toxicity limit. Math. Biosci., 100 (1990), 49-67.

[20] L. S. Pontryagin, V. G. Boltyanskii, R. V. Gamkrelidze, E. F. Mishchenko. The mathematical theory of optimal processes. Gordon and Breach, 1962.

[21] G. W. Swan, T. L Vincent. Optimal control analysis in the chemotherapy of IgG multiple myeloma. Bull. of Math. Bio., 39 (1977), 317-337.

[22] A. Swierniak, U. Ledzewicz, H. Schattler. Optimal control for a class of compartmental models in cancer chemotherapy. Int. J. Appl. Math. Comput. Sci., 13 (2003), No. 3, 357368.

[23] M. Villasana A. Radunskaya. A delay differential equation model for tumor growth. J. Math. Bio., 47 (2003), 270-294. 